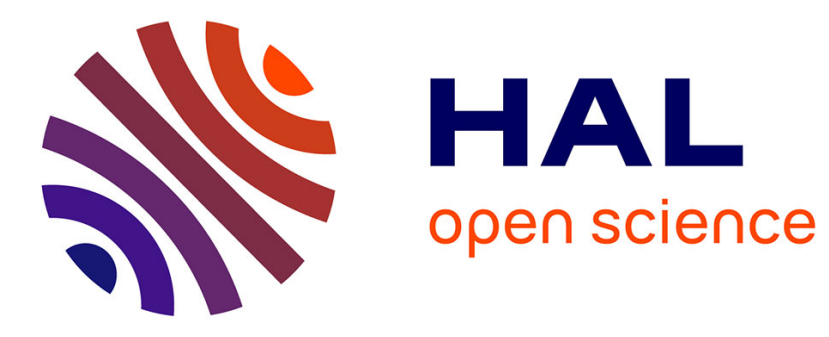

\title{
Mixing lamellae in a shear flow
}

Mathieu Souzy, Imen Zaier, Henri Lhuissier, Tanguy Le Borgne, Bloen Metzger

\section{To cite this version:}

Mathieu Souzy, Imen Zaier, Henri Lhuissier, Tanguy Le Borgne, Bloen Metzger. Mixing lamellae in a shear flow. Journal of Fluid Mechanics, 2018, 838, pp.R3. 10.1017/jfm.2017.916 . hal-01768670

\section{HAL Id: hal-01768670 \\ https://hal.science/hal-01768670}

Submitted on 1 Jun 2018

HAL is a multi-disciplinary open access archive for the deposit and dissemination of scientific research documents, whether they are published or not. The documents may come from teaching and research institutions in France or abroad, or from public or private research centers.
L'archive ouverte pluridisciplinaire HAL, est destinée au dépôt et à la diffusion de documents scientifiques de niveau recherche, publiés ou non, émanant des établissements d'enseignement et de recherche français ou étrangers, des laboratoires publics ou privés. 


\title{
Mixing lamellae in a shear flow
}

\author{
Mathieu Souzy ${ }^{2}$, Imen Zaier ${ }^{1}$, Henri Lhuissier ${ }^{1}$, Tanguy Le Borgne ${ }^{2} \&$ \\ Bloen Metzger ${ }^{1}$ \\ ${ }^{1}$ Aix-Marseille Université, IUSTI-CNRS UMR 7343, 13453 Marseille Cedex 13, France. \\ ${ }^{2}$ Geosciences Rennes, UMR 6118 Université Rennes 1 CNRS 35042 Rennes France
}

(Received 1 June 2018)

Mixing dynamics in flows are governed by the coupled action of diffusion and stretching by velocity gradients. This leads to the development of elongated lamellar structures in scalar fields where concentration fluctuations exist at scales set by the Batchelor scale. Because the latter is generally too small to be resolved experimentally, observation of these mechanisms remains an outstanding challenge. Here we present high resolution experiments allowing for the precise quantification of the evolution of concentration distributions at the scale of a single lamella experiencing diffusion, stretching and aggregation with other lamellae. Quantitative agreement is found with analytical predictions for the lamella's concentration profile, Batchelor time, Batchelor length-scale, and concentration distribution for a large range of Péclet numbers and without adjustable parameter. This benchmark configuration is used to set the experimental spatial-resolution required to quantify the concentration Probability Density Functions (PDF) of scalar mixtures in fluids. The diffusive coalescence of two nearby lamellae, the mechanism by which scalar mixtures ultimately reach uniformity, is shown to induce a complex transient evolution of the concentration PDFs.

\section{Introduction}

Mixing is a key process in many industrial applications and natural phenomena (Ottino 1989). Practical examples include glass manufacture, processing of food, micro-fluidic manipulations, and contaminant transport in the atmosphere, oceans and hydrological systems. During the last decades, substantial progresses have been made in the description of mixing in systems as complex as turbulent flows (Warhaft 2000, Shraiman \& Siggia 2000, Falkovich et al. 2001, Duplat \& Villermaux 2008, Kalda 2000), oceanic and atmospheric flows (Rhines \& Young 1983), the earth mantle (Allègre \& Turcotte 1986), porous media (Dentz et al. 2011, Villermaux 2012, Le Borgne et al. 2015) and sheared particulate suspensions (Souzy et al. 2017). In particular, the conceptualization of scalar mixtures as ensembles of 'lamellae' evolving through stretching, diffusion and aggregation (Ranz 1979, Villermaux \& Duplat 2003) has allowed deriving quantitative accurate theoretical predictions for the evolution of the full concentration Probability Density Functions (PDF) for a broad range of flows. Within this framework (valid for lamellae thinner than the smallest scale within the flow), mixing is driven by three processes occurring simultaneously: i) stretching a scalar field by a given flow creating elongated lamellar structures, ii) diffusion and compression that compete to define concentration gradients and iii) lamella coalescence leading ultimately to the final homogeneity of the system (Duplat \& Villermaux 2008).

In complex configurations such as those listed above, the evolution of the concentration distribution is sensitive to a number of characteristics of the system: the distribution of 
stretching rates, the macroscopic dispersion rate, the scalar molecular diffusion coefficient and the rate at which lamellae aggregate. While their influence on the dynamics of mixing is clear from a theoretical point of view, they can rarely be observed independently. Moreover, in spite of the numerous experimental studies on mixing, the question of the spatial resolution required to quantify the evolution of a concentration field has received little attention. This latter point is obviously crucial since under-resolved images, by artificially broadening the concentration distribution of isolated lamellae or conversely, by sharpening the concentration distribution of bundles of adjacents lamellae, lead to an erroneous appreciation of the mixing rate.

Here, we present highly resolved experiments where the basic mechanisms governing mixing are quantified individually: the formation of elongated lamellae by stretching, the establishment of a Batchelor scale by competition between compression and diffusion, the enhancement of diffusive mixing by stretching and the diffusive aggregation of lamellae. We consider for this a lamella formed by photo-bleaching of a fluorescent dye in a laminar shear flow where the concentration distribution can be quantified using a well controlled experimental set-up, built specifically to resolve small length scales. This benchmark experiment was chosen precisely because it is well established theoretically. Unambiguous conclusions can therefore be drawn regarding the spatial resolution required to capture the evolution of the concentration distribution of an isolated lamella, and more generally within any mixing protocols. Moreover, it illustrates with an unprecedented resolution and level of details the basic lamellar theories for mixing at the scale of a single lamella. Last, we investigate the coalescence of two nearby lamellae specifically focusing on its impact on the evolution of the concentration distribution. The theoretical prerequisites are recalled in $\S 2$. After presenting the experimental set-up in $\S 3$, the measurements are reported in $\S 4$. Conclusions are drawn in $\S 5$.

\section{Mixing in a laminar shear flow}

\subsection{General picture}

We consider a lamella of dye of length $l_{0}$ and half width $s_{0}$ initially positioned perpendicular to the flow as illustrated in Figure 1.a. As the lamella is advected by a laminar shear flow with a shear-rate $\dot{\gamma}$, its length increases as $l(t)=l_{0} \sqrt{1+(\dot{\gamma} t)^{2}}$. Thus, considering the sole effect of the advection field (for now neglecting that of molecular diffusion), the half width of the lamella $s_{A}(t)$ decreases following $s_{A}(t)=s_{0} / \sqrt{1+(\dot{\gamma} t)^{2}}$ (see Figure 1.b) since in this two dimensional flow, mass conservation prescribes $s_{0} l_{0}=s_{A}(t) l(t)$. The effect of the shear flow can thus be quantified by a compression rate $-1 / s_{A} d s_{A} / d t$ which describes how fast the lamella transverse dimension thins down owing to its stretching by the advection field. Conversely, molecular diffusion tends to broaden the lamella with a diffusive broadening rate $D_{0} / s_{A}^{2}$, where $D_{0}$ is the molecular diffusion coefficient of the scalar. Balancing these two rates, $-1 / s_{A} d s_{A} / d t \sim D_{0} / s_{A}^{2}$ and assuming $\dot{\gamma} t \gg 1$, naturally defines a time-scale, also called the Batchelor time, $t_{B} \sim P e^{1 / 3} / \dot{\gamma}$ where $P e=\dot{\gamma} s_{0}^{2} / D_{0}$ denotes the Péclet number. This time corresponds to the onset of the homogenization of the concentration levels within the system, beyond which the concentration levels within the lamella start to significantly decay.

\subsection{Exact solution}

The complete description of the evolution of the lamella of dye can be found by directly solving the full advection-diffusion equation 


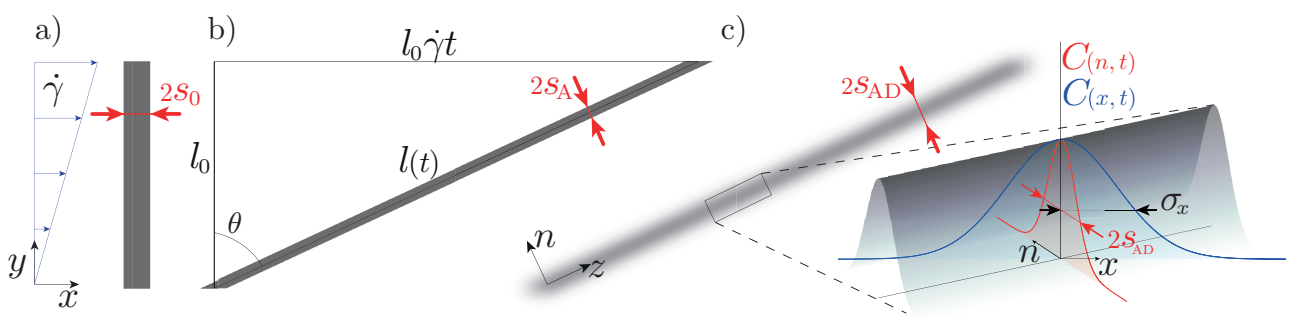

Figure 1. a) Schematic of a lamella of dye of initial length $l_{0}$ and half width $s_{0}$ advected in a laminar shear flow. b) Effect of the advection field alone: the strain $\dot{\gamma} t$ has stretched the lamella and thinned down its transverse dimension to $2 s_{\mathrm{A}}(t)$. c) Effect of both advection and molecular diffusion: at the same strain, the half width of the lamella is denoted $s_{\mathrm{AD}}(t)$. Inset: schematic of the gaussian concentration field of the lamella with its concentration profiles along the flow $C(x, t)$ and transverse to the lamella $C(n, t)$.

$$
\frac{\partial C}{\partial t}+\mathbf{u} \cdot \nabla C=D_{0} \nabla^{2} C
$$

where $\mathbf{u}=(\dot{\gamma} y, 0,0)$ denotes the advection field, and $C$ the concentration field.

This equation can be simplified (Ranz 1979, Rhines \& Young 1983, Ottino 1989, Meunier \& Villermaux 2003) if written in a moving frame $(n, z)$ aligned with the directions of maximal compression and stretching of the lamellae (see Figure 1.c), and by using Ranz's transform (Ranz 1979) which uses warped time units, $\tau=\int_{0}^{t} D_{0} / s_{A}^{2}\left(t^{\prime}\right) d t^{\prime}$, and distances normalized by the lamella transverse dimension, $\xi=n / s_{A}(t)$. Equation $(2.1)$ then reduces to a simple diffusion equation

$$
\frac{\partial C}{\partial \tau}=\frac{\partial^{2} C}{\partial \xi^{2}}
$$

whose solution for an initial gaussian concentration profile with maximum $C_{0}$ is

$$
C(\xi, \tau)=\frac{C_{0}}{\sqrt{1+4 \tau}} e^{-\frac{\xi^{2}}{1+4 \tau}} .
$$

The maximum concentration of the lamella thus decays with time according to

$$
C_{\text {max }}(t)=\frac{C_{0}}{\sqrt{1+4 \tau}} .
$$

Since the half width of the lamella in Ranz's units is $\sigma_{\xi}=(1+4 \tau)^{1 / 2}$ (see equation $2.3)$, the transverse dimension of the lamella $s_{\mathrm{AD}}(t)$, accounting both for the effect of advection and diffusion, and expressed in standard unit of length is

$$
s_{\mathrm{AD}}(t)=\sigma_{\xi} s_{\mathrm{A}}(t)=\frac{s_{0}(1+4 \tau)^{1 / 2}}{\sqrt{1+(\dot{\gamma} t)^{2}}} .
$$

The latter expression gives access to the half width of the lamella along the flow ( $x$-direction)

$$
\sigma_{x}(t)=\frac{s_{\mathrm{AD}}(t)}{\cos \theta}=\sigma_{0}(1+4 \tau)^{1 / 2},
$$

with $\sigma_{0}=s_{0}$ and $\cos \theta=1 / \sqrt{1+(\dot{\gamma} t)^{2}}$ (see Figure 1 ). 
We now have access to a more accurate estimation of the Batchelor time which by definition is reached when $\tau=1$ (Ranz 1979). Since $\tau=D_{0}\left(t+\dot{\gamma}^{2} t^{3} / 3\right) / s_{0}^{2}$, the Batchelor time, for $P e \gg 1$, is

$$
t_{\mathrm{B}} \approx \frac{(3 P e)^{\frac{1}{3}}}{\dot{\gamma}}
$$

At this time, the transverse dimension of the lamella, from now on referred to as the Batchelor scale (Batchelor 1959), is found to be

$$
s_{\mathrm{AD}}\left(t_{\mathrm{B}}\right)=s_{0} \sqrt{5}(3 P e)^{-\frac{1}{3}} .
$$

Last, the evolution of the concentration distribution $P(C, t)$ of the lamella can be derived. Using the following change of variables $P(C) d C=P(x) d x$ yields $P(C)=$ $P(x)|d C / d x|^{-1}$ which can easily be expressed from

$$
C(x, t)=C_{\max }(t) e^{-\frac{x^{2}}{\sigma_{0}^{2}(1+4 \tau)}},
$$

and the uniformity of $P(x)$. Considering the range of concentration $C_{\mathrm{th}} \leq C \leq C_{\max }(t)$, above any arbitrary threshold concentration $C_{\text {th }}$ (larger than the experimental background noise), one obtains

$$
P(C, t)=\frac{1}{\beta C \sqrt{\ln \left(C_{\max } / C\right)}},
$$

where $\beta$ is a normalizing prefactor insuring that $\int_{C_{\mathrm{th}}}^{C_{\max }(t)} P(C, t) d C=1$.

The above set of equations fully describes the evolution of a lamella of dye advected in a laminar shear flow.

\section{Experimental set-up}

The experimental set-up is shown in Figure 2. It consists of a shear-cell made of two parallel plexiglass plates sandwiching a fluid cell $85 \mathrm{~mm}$ long, $25 \mathrm{~mm}$ wide and of height $h=3 \mathrm{~mm}$. The fluid is sheared by setting the two plates into opposite motion with two high-resolution translation stages (not shown on the schematic). The travel-range of the plate enables a maximum strain of 30 . The cell is sealed on both ends by two PTFE plates (grey in Figure 2), and on the sides by two transparent side walls (not shown on the schematic) mounted on the bottom moving plate.

The fluid is a Newtonian mixture of triton X-100 (77.4 wt\%), zinc chloride (13.4 wt $\%$ ) and water $(9.2 \mathrm{wt} \%)$ having a density $\rho=1.19 \mathrm{~g} . \mathrm{cm}^{-3}$ and viscosity $\eta=4.2 \mathrm{~Pa} . \mathrm{s}$ which ensures laminar flow conditions (over the full range of shear rates investigated Re $=\rho \dot{\gamma} \mathrm{h}^{2} / \eta \leq 10^{-3}$ ). Prior to filling the cell, some fluorescent dye (Rhodamine 6G) is thoroughly mixed to the fluid at a concentration of $210^{-6} \mathrm{~g} \cdot \mathrm{mL}^{-1}$. The molecular diffusion coefficient of this dye was measured in the cell with the fluid at rest using a technic similar to that reported in Souzy et al. 2017. We find $D_{0} \approx 2.6610^{-13} \mathrm{~m}^{2} \mathrm{~s}^{-1}$ at a temperature of $22^{\circ} \mathrm{C}$.

The initial lamella is generated using fluorescent recovery after photo-bleaching, (Axelrod et al. 1976). A high-power-laser-diode beam (Laser Quantum Gem512-2.5W) is shaped into a thin laser sheet using the combination of lenses set in their 'bleaching' configuration as shown in Figure 2.a. This sheet, oriented in the $y-z$ plane (perpendicular to the flow) and used with full intensity, locally changes the conformation of the rhodamine molecules which irreversibly become unable to fluoresce.

Then, the set-up is set into its 'visualization' configuration by rotating the cylindrical 
a)

Long-distance microscope lens

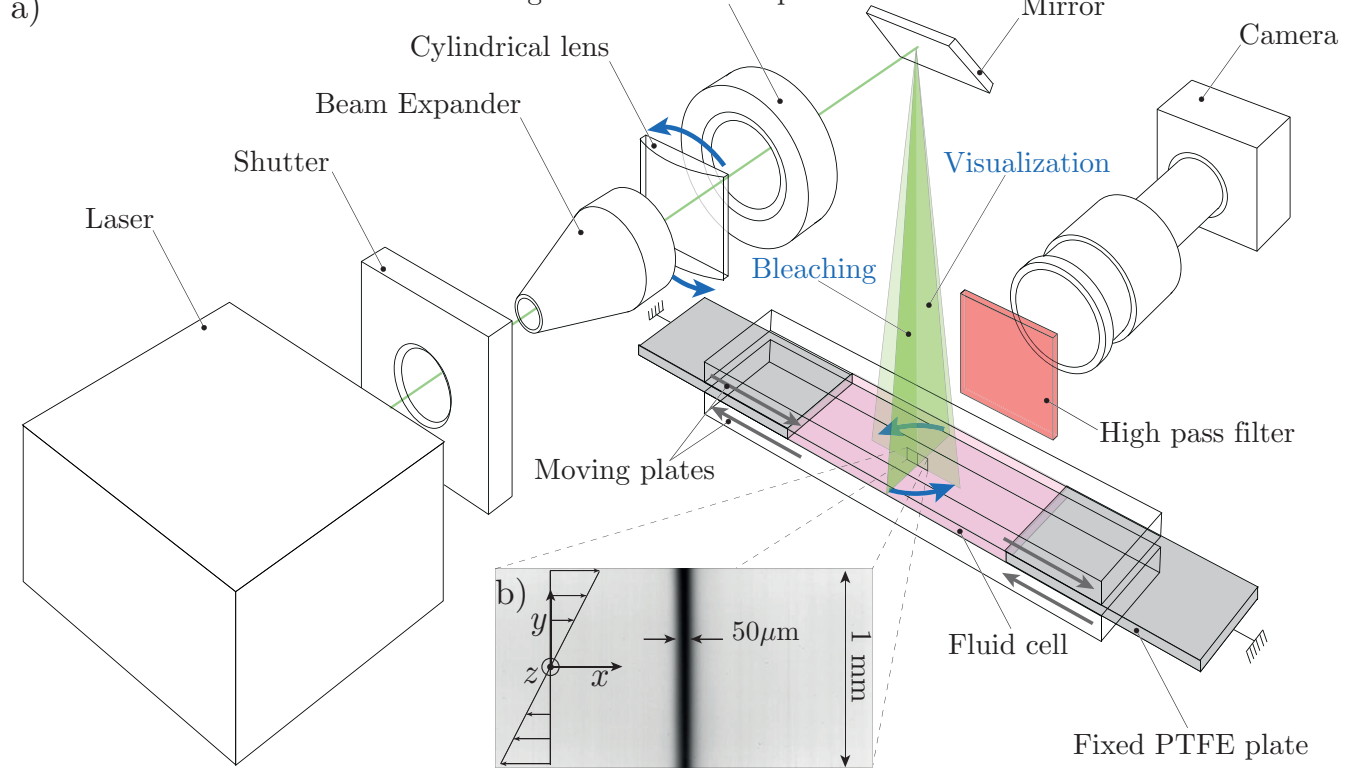

Figure 2. (color version online) a) Schematic of the set-up. b) Typical image of a lamella obtained by photo-bleaching.

lens $90^{\circ}$ : this orients the laser sheet along the $x-y$ plane (along the flow). The lamella then appears as a thin dark vertical line, see Figure 2.b. Note that the initial lamella is in fact a transverse sheet, rather than just a line. This avoids diffusion in the $z$-direction and enables us to study a purely $2 \mathrm{D}$ process. This photo-bleaching technic, relative to a direct injection of fluorescent dye, also insures well-controlled initial conditions: the lamella is uniform and its initial concentration profile is gaussian owing to the gaussian nature of the impacting laser beam.

Immediately after bleaching, the fluid is sheared at the desired shear rate and images of the evolution of the lamella are acquired using a camera (Basler Ace2000-50gm, $2048 \times 1080$ pixel $^{2}, 12$ bit) coupled to a high-resolution macroscope (Leica Z16 APO $\times 1$ ). The set-up was designed to provide an image resolution $(0.8 \mu \mathrm{m} /$ pixel $)$ small enough to resolve the Batchelor scale (equation 2.8). For instance, with $\dot{\gamma}=0.01 \mathrm{~s}^{-1}$ and $s_{0}=25 \mu \mathrm{m}$, we have $s_{\mathrm{AD}}\left(t_{B}\right)=s_{0} \sqrt{5}\left(3 \dot{\gamma} s_{0}^{2} / D_{0}\right)^{-\frac{1}{3}} \approx 10 \mu \mathrm{m}$. A high-pass filter $(590 \mathrm{~nm})$ is positioned between the sample and the camera to eliminate direct light reflexions. To avoid photobleaching during the image acquisition, the intensity of the laser is lowered to $100 \mathrm{~mW}$ and the image acquisition is synchronized with a shutter opening only during acquisition times. Note that all experiments are performed at $T=22 \pm 0.05^{\circ} \mathrm{C}$ by setting the temperature of the water running through the bottom moving plate with a cryo-thermostat.

\section{Experimental results}

\subsection{A single lamella}

Figure 3.a shows successive pictures of a lamella undergoing a laminar shear (see also supplementary movie 1). Initially vertical and highly contrasted, the lamella progressively tilts under the effect of the shear flow while blurring under the effect of molecular diffusion. Accurate measurement of the lamella's concentration profile along the flow $(x$-direction $)$ are obtained by averaging over all horizontal lines of pixels after trans- 
a)
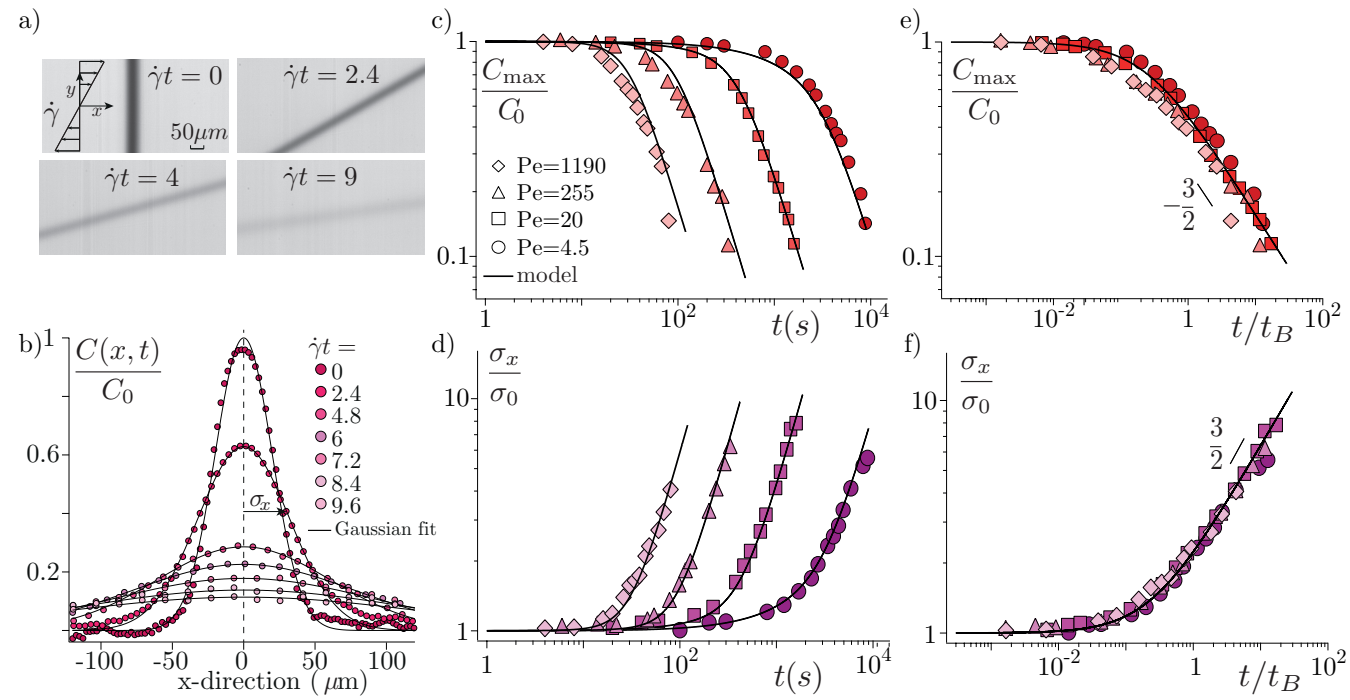

FiguRE 3. (color version online) a) Successive images of a lamella undergoing shear (see also supplementary movie 1). b) Corresponding averaged concentration profiles along the flow $(x$-direction). The black lines are fitting gaussian profiles. c) Normalized maximum concentration $C_{\max } / C_{0}$ and d) normalized half width of the concentration profiles $\sigma_{x} / \sigma_{0}$ versus time for experiments performed at different Péclet numbers. The black lines correspond to equation 2.4 in c) and equation 2.6 in d). In both cases, $\dot{\gamma}, s_{0}$ and $D_{0}$ are set and fixed by the experimental conditions. e) and f) Same data plotted versus $t / t_{B}$.

lating these lines to make their maximum concentration coincide. The resulting average concentration profile of the lamella is shown in Figure 3.b for successive strains: the maximum concentration decays while the width increases. These trends are well captured by fitting each concentration profile with a gaussian of the form $C(x, t)=C_{\max }(t) e^{-x^{2} / \sigma_{x}^{2}(t)}$ (see Figure 3.b).

The resulting maximum concentration $C_{\max }(t)$ and width $\sigma_{x}(t)$ are plotted in Figure 3.c and 3.d versus time for experiments performed at different Péclet number $(4.5 \leq$ $P e \leq 1190)$. The Péclet number was varied by repeating the experiment at various shear-rates $\dot{\gamma}=\left[6 \times 10^{-4}-0.3\right] \mathrm{s}^{-1}$. The agreement with equations 2.4 and 2.6 is very good for both $C_{\max }(t)$ and $\sigma_{x}(t)$. Note that in both cases, $\dot{\gamma}, s_{0}$ and $D_{0}$ are fixed by the experimental conditions; there is thus no adjustable parameter. When plotted as a function of the dimensionless time $t / t_{B}$, where $t_{B}=(3 \mathrm{Pe})^{1 / 3} / \dot{\gamma}$ is the Batchelor time, these data are found to collapse, for all Pe, on the same master curve, see Figure 3.e and f. For $t<t_{B}, C_{\max }$ and $\sigma_{x}$ remain constant. Then when the effect of molecular diffusion becomes significant, i.e for $t>t_{B}, C_{\max }$ (respectively $\sigma_{x}$ ) starts to decrease (respectively increase) following the power law $t^{-3 / 2}$ (respectively $t^{3 / 2}$ ), consistently with the long time trends of equations 2.4 and 2.6. These measurements clearly illustrate how mixing is accelerated by imposing an external macroscopic shear: larger applied shear rates (larger Péclet numbers) result in earlier mixing times.

We have so far probed the lamella along the direction of the flow. However, further insight into the mixing process, specifically on the advection-diffusion coupling presented above, are provided by probing the lamella width along its transverse direction (along $n$, see Figure 1). Figure 4.a shows the evolution of $s_{\mathrm{AD}}(t)$ measured experimentally. At an intermediate time, the thickness of the lamella is found to decrease like $t^{-1}$. After reaching a minimum, it increases like $t^{1 / 2}$. These trends precisely illustrate the expected 


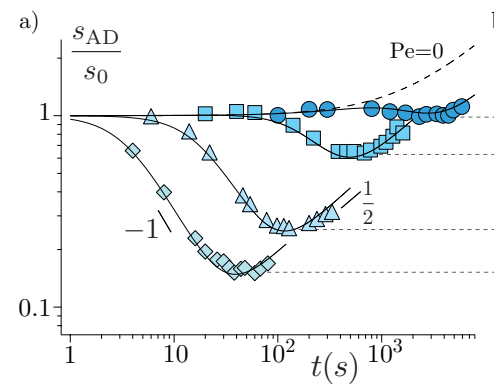

b)

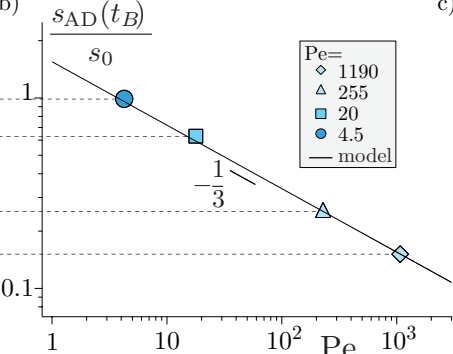

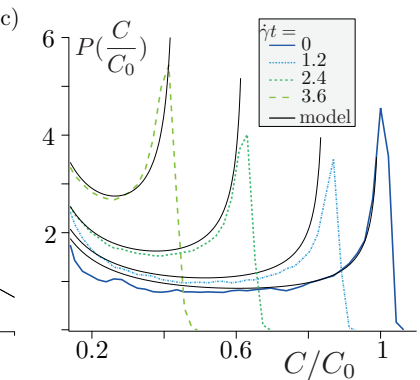

Figure 4. (color version online) a) Evolution of transverse dimension of the lamella $s_{\mathrm{AD}}(t) / s_{0}$ versus time for experiments performed at different Péclet numbers. The black lines correspond to equation 2.5. The dotted line corresponds to the solution in the absence of shear, i.e. in the pure diffusion limit $(\mathrm{Pe}=0)$. b) Corresponding Batchelor scale $s_{\mathrm{AD}}\left(t_{B}\right) / s_{0}$ versus Péclet number; the black line corresponds to equation 2.8. c) Concentration distribution $P\left(C / C_{0}\right)$ measured at successive strains, $\dot{\gamma} t$, for a lamella sheared at $P e=4.5$. The black lines correspond to equation 2.10 with $\dot{\gamma}$. In all cases, $\dot{\gamma}, s_{0}$ and $D_{0}$ are fixed by the experimental conditions.

interplay between advection and diffusion. The lamella width initially decreases as imposed by the kinematics of the flow following the intermediate time trend (for $t<t_{B}$ ) of equation 2.5, $s_{\mathrm{AD}}(t) \sim s_{0}(\dot{\gamma} t)^{-1}$. However, this compression of the lamella progressively steepens its concentration gradients which beyond the Batchelor time, eventually makes the broadening effect due to molecular diffusion become dominant. The transverse dimension of the lamella then re-increases diffusively like $t^{1 / 2}$. At the Batchelor time $t_{B}$, the lamella typically reaches its minimum thickness which is equal to the Batchelor scale $s_{\mathrm{AD}}\left(t_{B}\right)$ (within $3 \%$ ). As shown in Figure 4.b, reporting this direct measurement of the Batchelor scale obtained for various Péclet numbers matches the expected prediction $s_{\mathrm{AD}}\left(t_{\mathrm{B}}\right)=s_{0} \sqrt{5}(3 P e)^{-\frac{1}{3}}$, see equation 2.8 .

To fully describe the mixing process, we also measure the evolution of the lamella's concentration distribution $P(C, t)$. The distribution $P(C)$ is obtained from the histogram of intensities collected from all the pixels constituting the lamella which are above a threshold $C_{\mathrm{th}}=0.1 C_{0}$. This discards the background image noise enabling us to focus on the peak of interest, that of high concentration. Changing the value of the threshold above the background noise, changes the extend of the spatial domain over which $P(C)$ is computed, but it does not affect its shape. We obtain concentration distributions which have a characteristic U-shape (Ottino 1989), see Figure 4.c. The distribution's maximum probability, initially located at $C=C_{0}$, progressively drifts to lower values as the lamella is stretched and diffuses. The prediction provided by equation 2.10 is in good agreement with the measured concentration distributions obtained for all Péclet number and again, without adjustable parameter.

The distributions shown above are well resolved since the Batchelor scale is always larger than 10 pixels. However, in most studies on mixing, such a large resolution is not achieved. Moreover, when mixing is investigated in complex systems such as turbulent flows (Duplat \& Villermaux 2006), porous media (Le Borgne et al. 2015) or recently in sheared particulate suspensions (Souzy et al. 2017), the initial lamella of dye does not evolve towards a single lamella with uniform thickness but instead, towards a large number of lamellae having widely distributed thicknesses. In such situations, it is important to know which part of the distribution of lamellae can be resolved experimentally.

To address this point, we systematically investigate how the set-up spatial resolution can bias the measured concentration distribution of a single lamella. This can be achieved by a simple coarsening protocol: the reference concentration distribution (blue dotted line 


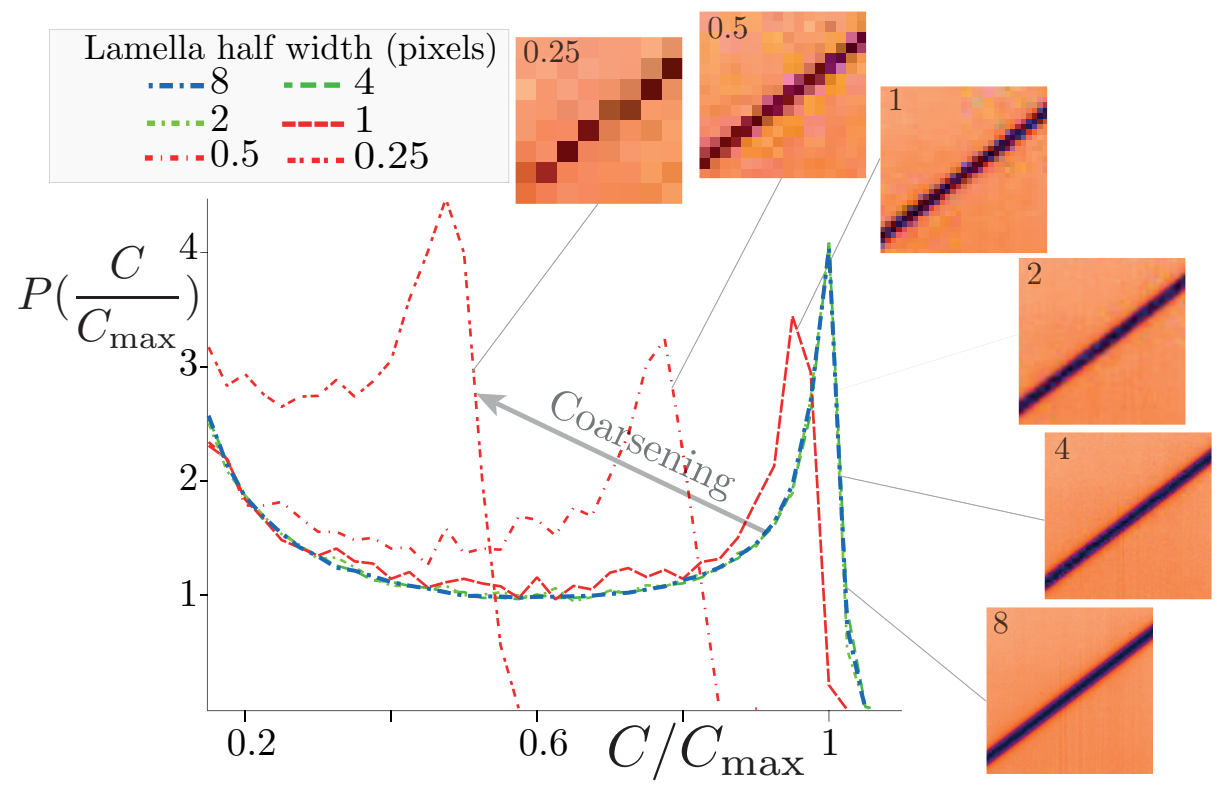

Figure 5. (color version online) Distributions of concentration $P\left(C / C_{\max }\right)$ obtained from the progressive digital coarsening of an experimental image.

in Figure 5) is obtained from a highly resolved image where the lamella half width spans 8 pixels. When coarsening the pixels of this reference image, (merging $2 \times 2$ pixels into one, $4 \times 4$ pixels into one and so on) we find that the concentration distribution remains unchanged for images having 4 and 2 pixels across the lamella half width (green dotted lines in Figure 5). Conversely, larger coarsening, i.e. images having 1 pixels or less across the lamella half width, yield erroneous results: the concentration distribution departs from the reference one (red dotted lines in Figure 5). The limit of 1 pixels per lamella half width is surprisingly small and comes as encouraging news for experimentalists. This limit holds as long as the concentration profile is smooth (e.g gaussian) and there is a finite tilt between the lamella and the lines of pixels. In such case, the progressive drift of the lamella position relative to that of the pixels scans all the possible concentration levels, thereby providing results consistent with the fully resolved PDF.

\subsection{Two Lamellae}

As mentioned in the introduction, mixing in complex systems generally involves coalescence between dispersed lamellae. This latter step is crucial for the system to reach its final homogeneity. Here, the photo-bleaching technic is used to produce two adjacent lamellae and investigate a single coalescence event.

Figure 6.a shows images of two parallel lamellae captured at successive strains (see also supplementary movie 2). The two lamellae are initially distinct from each other and, as they are stretched by the flow and diffuse, they progressively merge to eventually form one single entity. The evolution of their concentration profiles measured along the flow $x$-direction are shown in Figure 6.b. The two maxima of concentration, corresponding to each lamella, decay (blue arrows) while the minimum located in between increases (green arrow). This goes on until the two lamellae merge into one single lamella whose maximum subsequently decreases (yellow arrow). This evolution can easily be predicted owing to the linearity of the diffusion equation: the concentration profile of a set of two lamellae, with individual profiles $C_{1}(x, t)$ and $C_{2}(x, t)$, is simply obtained from the 
a)

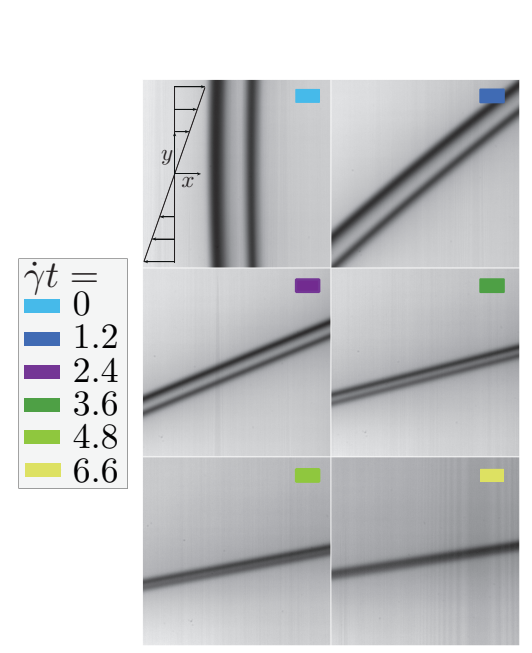

b)
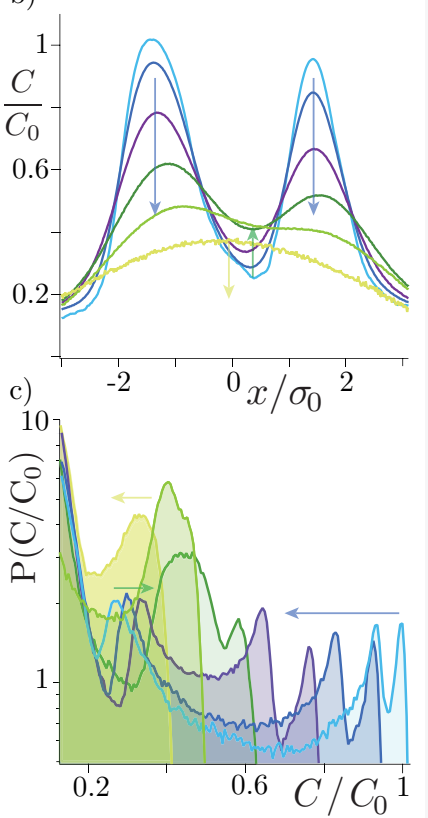

d)
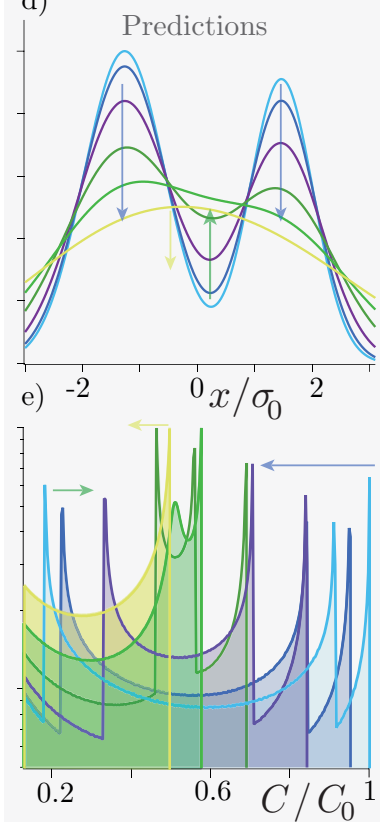

FiguRE 6. (color version online) a) Coalescence of two nearby lamellae advected in a laminar shear flow (see also supplementary movie 2). Evolution of the corresponding experimental b) concentration profiles and c) concentration distributions obtained at successive strains $\dot{\gamma} t$. Predictions for the evolution of d) the concentration profiles and e) the concentration distributions at the same strains. Again, $\dot{\gamma}, s_{0}$ and $D_{0}$ are fixed by the experimental conditions.

summation $C(x, t)=C_{1}(x, t)+C_{2}(x, t)$ (Fourier 1822). Using, for each lamella, equation 2.9 with the initial experimental maximum intensity and width, we thereby obtain the concentration profiles shown in Figure 6.d.

To anticipate the impact of this coalescence on the evolution of the concentration distribution, lets recall that $P(C) \sim|d C / d x|^{-1}$. Thus, each value of $C(x, t)$ where the concentration profile presents an horizontal slope gives rise to a peak in the concentration distribution. This can be clearly observed on the experimental concentration distributions shown in Figure 6.c. As long as the two lamellae are distinct, one not only observes two peaks at large concentrations (corresponding to the maximum of concentration of each lamellae), but also one peak at small concentration (corresponding to the minimum concentration located in between the two lamellae). Then, as the lamellae are stretched and diffuse, the two peaks corresponding to the two concentration maxima, move to the left (blue arrow in Figure 6.c). Conversely, as the concentration in between the two lamellae increases, the small concentration peak moves to the right (green arrow in Figure 6.c. Coalescence occurs when the three peaks collide; the distribution eventually recovers its characteristic U-shape as the lamellae have coalesced into a single entity. The same phenomenology can be observed on the concentration distributions obtained from numerically computing the slope of the predicted concentration profiles, see Figure 6.e.

\section{Discussion and conclusions}

We have explored experimentally the basic mechanisms of mixing in stirred flows by thoroughly investigating the evolution of an initially segregated scalar (a lamella of flu- 
orescent dye) stretched within a laminar shear flow. A high resolution set-up, using a photo-bleaching technic to generate and control the shape of the initial lamella, was built in order to resolve length scales at which diffusion plays a significant role. Our measurements of the evolution of the lamella concentration profiles and concentration distributions are, without adjustable parameter, in excellent quantitative agreement, for $P e=[4.5-1190]$, with the theoretical predictions.

We also investigated the evolution of the lamella's transverse dimension which conspicuously illustrates the advection-diffusion coupling yielding at intermediate time to a $t^{-1}$ compression of the lamella dominated by the kinematics of the flow, followed, after the Batchelor time, by a $t^{1 / 2}$ broadening dominated by molecular diffusion. The Batchelor scale, which to our knowledge was experimentally observed only once (Meunier et al. 2015), was here measured systematically for various Péclet number and found to follow the expected behavior, $s_{\mathrm{AD}}\left(t_{B}\right) / s_{0}=\sqrt{5}(3 P e)^{-\frac{1}{3}}$.

Most importantly, through a coarsening protocol, we determined the minimal experimental spatial resolution required to resolve the concentration distribution of a single lamella: its half width must be larger than 1 pixel. This requirement is general and constrains the measurement of any mixing protocol. Indeed, for all stretching protocol, lamellae reach their minimum thickness at the Batchelor time while they are still isolated individual entities. Resolving $P(C)$ at this time, which requires to resolve each individual lamella, therefore is the most demanding in term of spatial resolution: the half width of the lamella at the Batchelor time, $s_{\mathrm{AD}}\left(t_{B}\right)$, shall at least span 1 pixel. Note that measuring $\mathrm{P}(\mathrm{C})$ at longer times can be less stringent. Indeed, beyond the Batchelor time, lamellae diffuse sufficiently that, when their spacing becomes sufficiently small, nearby lamellae start merging together. The concentration gradients then no longer vary over the lamellae transverse dimension but instead, over a larger length-scale which reflects the size of the bundles of merging lamellae. In the context of turbulent mixtures for instance, this 'coarse-grained scale' was proposed to follow $\eta=L S c^{-2 / 5}$ where $L$ denotes the stirring length and $S c$ the Schmidt number (Duplat \& Villermaux 2006). However, the latter length-scale is only relevant after lamellae have merged which occurs after the Batchelor time. Therefore, resolving $\mathrm{P}(\mathrm{C})$ at all times requires an experimental spatial resolution satisfying $s_{\mathrm{AD}}\left(t_{B}\right)>1$ pixel.

Finally, we have investigated the coalescence between two lamellae and its impact on the evolution of the concentration distribution. Lamellae's overlap gives rise to a nontrivial peak at low concentration. This observation is important as it may be relevant to interpret more complex situations.

To conclude, the high resolution experimental technics developed for the present study and the determination of its limitations, open promising perspectives for future studies on mixing.

We thank E. Villermaux and P. Meunier for their inspiring comments and suggestions. We would also like to thank Sady Noel for machining the experimental set-up. This work was supported by ANR JCJC SIMI 9, by the Labex MEC ANR-10-LABX-0092, by ANR-11-IDEX-0001-02 and by ERC consolidator grant ReactiveFronts 648377 .

\section{REFERENCES}

Allègre C. J., \& Turcotte D. L. 1986 Implications of a two-component marble-cake mantle Nature 323, 123-127.

Axelrod D., Koppel D. E., Schlessinger J., Elson E., \& Webb W. W. 1976 Mobility measurement by analysis of fluorescence photobleaching recovery kinetics Biophysical journal 16, 1055-1069. 
BAtChelor G. K. 1959 Small-scale variation of convected quantities like temperature in a turbulent fluid. part 1. general discussion and the case of small conductivity, J. Fluid Mech., 5, 113-133.

Dentz, M., LeBorgne, T., Englert, A. \& Bijeljic, B. 2011 Mixing, spreading and reaction in heterogeneous media: A brief review. J. Cont. Hydrol. 120-121, 1-17.

Villermaux E., \& Duplat, J. 2006 Coarse grained scale of turbulent mixtures Physical review letters, 97144506.

Duplat, J. \& Villermaux, E. 2003 Mixing by random stirring in confined mixtures J. Fluid Mech., 617 pp 51-86.

Falkovich, G., Gawedzki, K. \& Vergassola, M. 2001 Particles and fields in fluid turbulence. Rev. Mod. Phys. 73 (4), 913-975.

Fourier, J. 1822 Théorie Analytique de la Chaleur, Firmin Didot.

KAldA, J. 2000 Simple model of intermittent passive scalar turbulence. Phys. Rev. Lett. 84 (3), 471-474.

Le Borgne T., Dentz M. \& Villermaux E. 2015 The lamellar description of mixing in porous media, J. Fluid Mech. 770, 458-498.

Meunier P. \& Villermaux E. 2003 How vortices mix, J. Fluid Mech. 476, 213-222.

Meunier P. \& Villermaux E. 2010 The diffusive strip method for scalar mixing in two dimensions. J. Fluid Mech. 662, 134-172.

Meunier P., Huck P., Nobili C.,\& Villermaux E. 2015 Transport and diffusion around a homoclinic Point, In Chaos, Complexity and Transport: Proceedings of the CCT $15 \mathrm{pp}$. 152-162.

Oтtino J.M. 1989 "The Kinematics of Mixing: Stretching, Chaos, and Transport", Cambridge University Press.

RANZ W. E. 1979 Applications of a stretch model diffusion, and reaction in laminar and turbulent flows, AlChE Journal 25, 1.

Rhines, P. B., \& Young, W. R. 1983 How rapidly is a passive scalar mixed within closed streamlines?, Journal of Fluid Mechanics 133, 133-145.

Shraiman, B. I. \& Siggia, E. D. 2000 Scalar turbulence. Nature 405, 639-646.

Souzy M., Lhuissier H. , Villermaux E., \& Metzger B. 2017 Stretching and mixing in sheared particulate suspensions J. Fluid Mech. 812, 611-635.

Villermaux, E. \& Duplat, J. 2003 Mixing as an aggregation process. Phys. Rev. Lett. 91 (18), 184501-1-4.

Villermaux, E. 2012 Mixing by porous media Comptes Rendus Mcanique 340, 933-943.

Warhaft, Z. 2000 Passive scalars in turbulent flows Annu. Rev. Fluid Mech. 32, 203-240. 